\title{
MCR-NET: A MULTI-STEP CO-INTERACTIVE RELATION NETWORK FOR UNANSWERABLE QUESTIONS ON MACHINE READING COMPREHENSION
}

\author{
Wei Peng ${ }^{1,2}$, Yue $\mathrm{Hu}^{1,2 *}$, Jing $\mathrm{Yu}^{1,2}$, Luxi Xing ${ }^{1,2}$, Yuqiang Xie ${ }^{1,2}$, Zihao Zhu ${ }^{1,2}$, Yajing Sun ${ }^{1,2}$ \\ ${ }^{1}$ Institute of Information Engineering, Chinese Academy of Sciences, China \\ ${ }^{2}$ School of Cyber Security, University of Chinese Academy of Sciences, China
}

\begin{abstract}
Question answering systems usually use keyword searches to retrieve potential passages related to a question, and then extract the answer from passages with the machine reading comprehension methods. However, many questions tend to be unanswerable in the real world. In this case, it is significant and challenging how the model determines when no answer is supported by the passage and abstains from answering. Most of the existing systems design a simple classifier to determine answerability implicitly without explicitly modeling mutual interaction and relation between the question and passage, leading to the poor performance for determining the unanswerable questions. To tackle this problem, we propose a Multi-Step Co-Interactive Relation Network (MCR-Net) to explicitly model the mutual interaction and locate key clues from coarse to fine by introducing a co-interactive relation module. The co-interactive relation module contains a stack of interaction and fusion blocks to continuously integrate and fuse history-guided and current-query-guided clues in an explicit way. Experiments on the SQuAD 2.0 and DuReader datasets show that our model achieves a remarkable improvement, outperforming the BERT-style baselines in literature. Visualization analysis also verifies the importance of the mutual interaction between the question and passage.
\end{abstract}

Index Terms- Machine Reading Comprehension, Unanswerable Question, Co-Interactive Relation Module

\section{INTRODUCTION}

ПMachine Reading Comprehension (MRC) is a long-term goal in natural language processing, which aims to teach machines

\footnotetext{
${ }^{*}$ Corresponding author. E-mail: huyue@iie.ac.cn

Copyright 2021 IEEE. Published in ICASSP 2021 - 2021 IEEE International Conference on Acoustics, Speech and Signal Processing (ICASSP), scheduled for 6-11 June 2021 in Toronto, Ontario, Canada. Personal use of this material is permitted. However, permission to reprint/republish this material for advertising or promotional purposes or for creating new collective works for resale or redistribution to servers or lists, or to reuse any copyrighted component of this work in other works, must be obtained from the IEEE. Contact: Manager, Copyrights and Permissions / IEEE Service Center / 445 Hoes Lane / P.O. Box 1331 / Piscataway, NJ 08855-1331, USA. Telephone: + Intl. 908-562-3966.
}

Article: Endangered Species Act

Passage: ... Other legislation followed, including the Migratory Bird Conservation Act of 1929 ... These later laws had a low cost to society - the species were relatively rare-and little opposition was raised.

Question: Which laws faced significant opposition?

Baseline (plausible answer): Later laws.

MCR-Net: None.

Fig. 1. An unanswerable question in SQuAD 2.0, along with plausible (but incorrect) answers. Relevant clues are shown in blue.

to understand the given passage and answer questions [1]. A machine reading comprehension system should not only answer questions when possible, but also determine when no answer is supported by the paragraph and abstain from answering. In the extractive MRC task, most of methods [2, 3, 4] make an assumption that the correct answer is guaranteed to exist in the context passage. Therefore, models only need to select the span that seems most related to the question, ignoring to check whether the answer is actually entailed by the text. So they are still far from true language understanding and are not capable of determining unanswerable questions [5, 6], which is much more challenging in practice.

In recent years, some initial work [7, 8] include that of which uses a classifier with a single fully connected layer to predict the probability of unanswerable questions. However, these studies lacks the explicit process of interaction. [9] proposes a verifiable system to verify if the candidate answer is actually supported by its surrounding sentence. However, the model uses the plausible answer which may be wrong to make an inference. The above methods lack precise positioning and analysis of the key clues that would be helpful to predict unanswerable questions. Referring to the example in Fig. 1, later laws and little opposition are key clues in the passage, model can reject to answer the question if it can locate the key clue little opposition and then infer that little opposition is conflict with significant opposition in the question. In this paper, we propose a Multi-Step Co-Interactive Relation Network (MCR-Net) which explicitly models the mutual interaction between the question and passage and locates key 


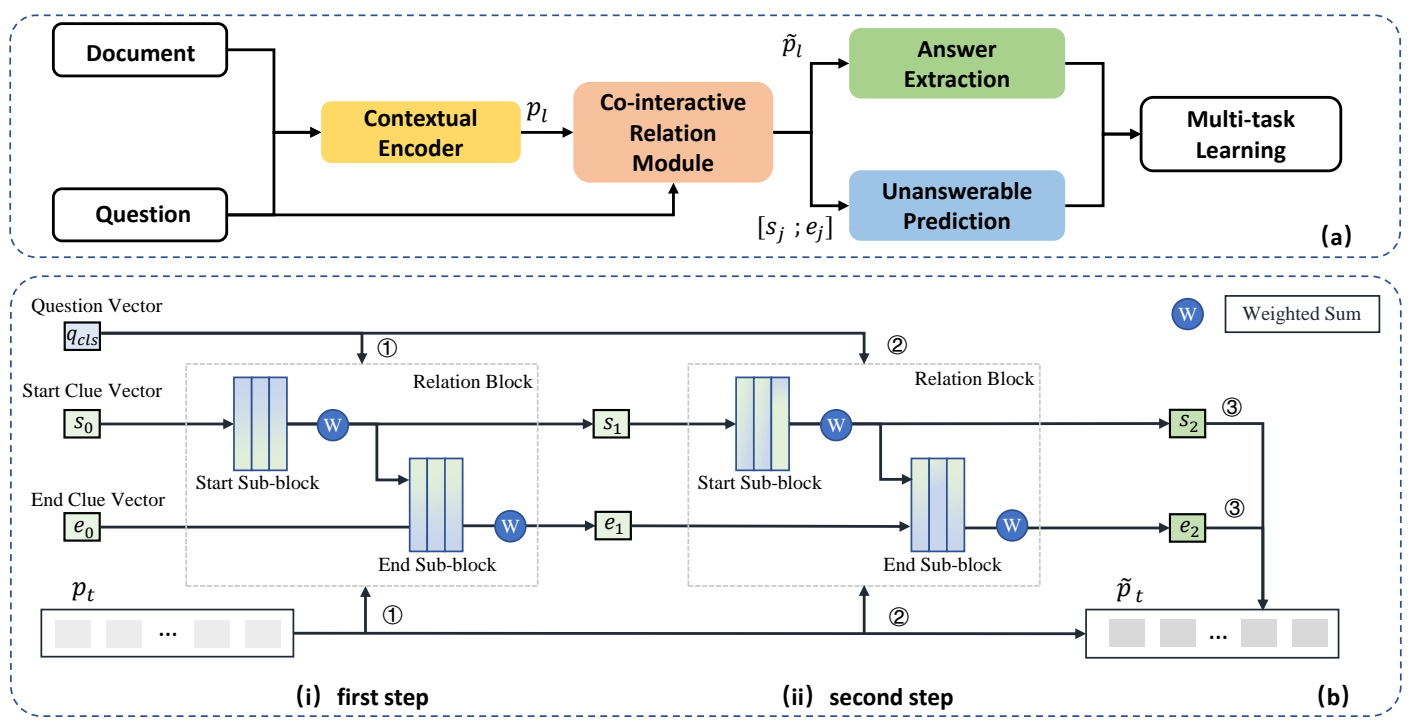

Fig. 2. (a) is the overview of our MCR-Net which consists of the Contextual Encoder, Co-Interactive Relation Module and Answer Predictor. (b) illustrates the details of the Co-Interactive Relation Module. (n) indicates the order of processes.

clues from coarse to fine for determining unanswerable questions. Specifically, we first consider the pre-trained language model as an encoder to obtain contextual representations of the question and passage. Then, the representations are fed into the Co-Interactive Relation Module to locate key clues for subsequent answer predictor. The important observation is the significant performance obtained from MCR-Net; it not only achieves the high performance on two datasets but also improves the Recall of unanswerable questions.

\section{METHODOLOGY}

As shown in Fig. 2 (a), the proposed model consists of three modules. First, the Contextual Encoder obtains the contextual representations of passage and question by pre-trained language model [10, 11, 12] such as RoBERTa and ALBERT. Then, the Co-Interactive Relation Module explicitly models the mutual interaction between the question and passage and locate key clues from coarse to fine. Finally, precise clues are utilized in Answer Predictor to make subsequent predictions.

\subsection{Contextual Encoder}

Considering the strong performance of pre-trained language models, we use them as the Contextual Encoder. Given a question $X^{Q}=\left\{x_{0}^{q}, \ldots, x_{M-1}^{q}\right\}$ and a passage $X^{P}=$ $\left\{x_{0}^{p}, \ldots, x_{N-1}^{p}\right\}$, following the BERT [13], the total length of the input is $L=(M+N+3), M$ and $N$ are the length of the passage and question. To reread the question to locate question-relevant clues, we obtain the representation of the pure question $q_{c l s}$ for a special token $[C L S]$ in Eqn.2, as:

$$
\begin{gathered}
p_{i}=\operatorname{BERT}\left([C L S], x_{i}^{q},[S E P], x_{i}^{p},[S E P]\right) \\
q_{j}=\operatorname{BERT}\left([C L S], x_{j}^{q},[S E P]\right)
\end{gathered}
$$

\subsection{Co-Interactive Relation Module}

The proposed Co-Interactive Relation Module is responsible for explicitly modeling the mutual interaction and relation information between the question and passage and capturing more accurate question-relevant clues. Specifically, it continuously integrates and fuses history-guided mutual relations and current-query-guided clues in each step of the interaction. Relation Block. As shown in Fig. 22(b), the module contains a stack of Relation Blocks, each of which can be stacked to perform multi-step interaction for better utilizing the mutual relation and knowledge. Motivated by pointer network[14], the blocks consist of two sub-blocks which interact together to obtain clues fragments. And the calculation of the end subblock considers the result of the start sub-block. $p_{l}$ and $q_{c l s}$ are regarded as the information flow of the module to gather information from the question and passage multiple times. $s_{j}$ and $e_{j}$ are the start and end clue vectors in the $j^{\text {th }}$ interactive step. The clue vectors fuse all possible clues based on relevance to the question. When $j=0$, we use a randomly initialized vector $s_{0}$ and $e_{0}$ as start and end clue vectors.

In the Start Sub-block, the module first concatenates $s_{j}$ and $p_{t}$ to enhance the representation of the passage $p_{t}$, as:

$$
\hat{p}_{t}^{j}=\operatorname{ReLU}\left(\operatorname{Linear}\left(\left[s_{j} ; p_{t}\right]\right)\right)
$$

where $j=\{0,1,2 \ldots\}$ means the $j^{t h}$ interactive step, [;] is vector concatenation across row. 
To obtain the question-relevant clues, the module rereads the question by using $q_{c l s}$. Then it computes a start probability distribution $\alpha^{j}$, as:

$$
\alpha_{t}^{j}=\operatorname{Softmax}\left(q_{c l s}^{\top} \hat{p}_{t}^{j}\right)
$$

where $\alpha_{t}^{j} \in \mathbb{R}^{L}$, indicating what information the question is more concerned about.

Given the $\hat{p}_{t}^{j}$ in Eqn. 3 and $\alpha^{j}$ in Eqn. 4, the weighted sum operation will be utilized to obtain the updated start clue vector $s_{j+1}$ which is a guider for the latter interactive.

$$
s_{j+1}=\sum_{t} \alpha_{t}^{j} \hat{p}_{t}^{j}
$$

After calculating the probability distribution $\alpha_{t}^{j}$ and the clue vector $s_{j}$ of the start position, we consider the End Subblock. Because of the temporal relationship, we introduce $s_{j}$ into the calculation of $e_{j+1}$ to further capture the mutual information. Similarly, the ending probability distribution $\beta^{j}$ and the end clue vector $e_{j}$ are yielded as:

$$
\begin{gathered}
\hat{p}_{t}^{j}=\operatorname{ReLU}\left(\operatorname{Linear}\left(\left[s_{j+1} ; e_{j} ; p_{t}\right]\right)\right) \\
\beta_{t}^{j}=\operatorname{Softmax}\left(\operatorname{Linear}\left(q_{c l s}^{\top} \hat{p}_{t}^{j}\right)\right) \\
e_{j+1}=\sum_{t} \beta_{t}^{j} \hat{p}_{t}^{j}
\end{gathered}
$$

where $s_{j+1}, e_{j} \in \mathbb{R}^{h}$ and $\beta^{j} \in \mathbb{R}^{L}$.

To further interact and locate the key clues from coarse to fine, the model iteratively updates the start and end clues vectors $s_{j-1}, e_{j-1}$. The $s_{j-1}$ and $e_{j-1}$ of the current block are regarded as the initial states of the subsequent block. Circularly, final states $s_{j}$ and $e_{j}$ which fuse the mutual interaction and related information are utilized to better capture the semantic relatedness, as $\tilde{p_{t}}=W_{g}^{\top}\left[p_{t} ; s_{j} ; e_{j}\right]$.

\subsection{Answer Predictor}

Answer Possibility Classifier. In order to enable the model to determine whether the question can be answered based on key clues, we utilize $s_{j}$ and $e_{j}$ for binary classification, which is different from previous methods. It is unanswerable when the score exceeds a threshold.

$$
\operatorname{score}_{i}=W_{s}^{\top}\left[s_{j} ; e_{j}\right], \quad \hat{s}_{i}=\sigma\left(\text { score }_{i}\right)
$$

where $W_{s} \in \mathbb{R}^{2 h}, \hat{s}_{i}$ is a scalar which means the unanswerable score of the $i^{t h}$ question.

The average cross-entropy loss among all the questions is optimized as:

$$
\mathcal{L}_{a n s}=-\frac{1}{N} \sum_{i=1}^{N}\left[\hat{y} \log \hat{s}_{i}+(1-\hat{y}) \log \left(1-\hat{s}_{i}\right)\right]
$$

where $N$ is the total number of the questions, $\hat{y} \in\{0,1\}$ denotes a label, $\hat{y}=1$ means the $i^{\text {th }}$ question is unanswerable.

\begin{tabular}{lcc}
\hline Model & Exact Match(\%) & F1(\%) \\
\hline DocQA [8] & 61.90 & 64.80 \\
Unet|16] & 70.30 & 74.00 \\
RMR + Answer Verifier [9] & 72.30 & 74.80 \\
Relation Network [17] & 79.20 & 82.60 \\
SG-Net [18] & 85.10 & 87.90 \\
\hline ALBERT-base & 76.05 & 80.07 \\
MCR-Net-base (ours) & 78.25 & 81.47 \\
\hline ALBERT-xxlarge & 84.78 & 88.08 \\
MCR-Net-xxlarge (ours) & $\mathbf{8 5 . 6 3}$ & $\mathbf{8 8 . 7 2}$ \\
\hline Human Performance & 86.30 & 89.00 \\
\hline
\end{tabular}

Table 1. Exact Match (EM) and F1 scores (\%) on SQuAD 2.0 development set for single model.

\begin{tabular}{lcc}
\hline Model & ROUGE-L(\%) & BLEU4(\%) \\
\hline Match-LSTM [2] & 39.00 & 31.80 \\
BiDAF [3] & 39.20 & 31.90 \\
V-Net [19] & 44.18 & 40.97 \\
\hline RoBERTa-base & 48.35 & 47.00 \\
MCR-Net-base (ours) & 50.16 & 48.64 \\
\hline RoBERTa-large & 49.82 & 48.22 \\
MCR-Net-large (ours) & $\mathbf{5 0 . 8 2}$ & $\mathbf{4 9 . 1 8}$ \\
\hline Human Performance & 56.10 & 57.40 \\
\hline
\end{tabular}

Table 2. Performance on the DuReader 2.0 test set.

Answer Extractor. Following [15], we consider $\tilde{p_{0}}$ as a sentinel to indicate the question is unanswerable. The answer span is calculated by predicting the start and the end indices. We compute the final probability $\alpha^{j}$ and $\beta^{j}$ of each word to be the start or end position, as:

$$
\gamma_{t}^{j}=\operatorname{Softmax}\left[\left(W_{c}^{\top} \tilde{p_{t}}\right)\right], \quad \eta_{t}^{j}=\operatorname{Softmax}\left[\left(W_{e}^{\top} \tilde{p_{t}}\right)\right]
$$

where $W_{c}, W_{e} \in \mathbb{R}^{h} \cdot \gamma^{j}, \eta^{j} \in \mathbb{R}^{L} \cdot \gamma_{0}^{j}$ and $\eta_{0}^{j}$ denote the score of sentinel that hints non-answerability.

The $\mathcal{L}_{\text {span }}$ loss function is to minimize the sum of the negative $\log$ probabilities of the true start and end positions. $s$ and $e$ represent the true start and end positions of the answer.

$$
\mathcal{L}_{\text {span }}=-\frac{1}{N} \sum_{i}^{N} \log \left(\gamma_{s}^{j}\right)+\log \left(\eta_{e}^{j}\right)
$$

\subsection{Joint Learning}

We combine the above two loss functions as the training loss:

$$
\mathcal{L}(\theta)=\lambda_{1} \mathcal{L}_{\text {span }}+\lambda_{2} \mathcal{L}_{\text {ans }}
$$

where $\theta$ is the all learnable parameters, and $\lambda_{1}$ and $\lambda_{2}$ are two hyper-parameters for controlling the weight of the rest tasks.

\section{EXPERIMENTS}

\subsection{Experimental Setting}

Datasets \& Evaluation Metrics. SQuAD2.0 [20] (contained 150k questions) and DuReader [6] (contained 300k questions) datasets provide unanswerable questions which contain 


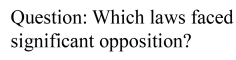

(a) first step

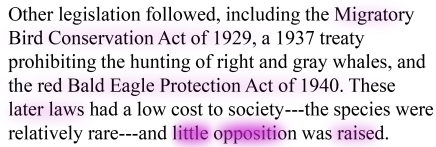

(b) second step

Fig. 3. The two subplots show the attention $(\alpha$ and $\beta$ ) of the first step and second step in the co-interactive relation module respectively. Apparently, the keywords little opposition in the passage is highlighted in (b), and the later laws is ignored in the second step, which is exactly the wrong answer.

\begin{tabular}{l|lccc}
\hline & Model & F1(\%) & R(\%) & P(\%) \\
\hline \multirow{2}{*}{ SQuAD 2.0 } & ALBERT-base & 82.37 & 77.00 & $\mathbf{8 8 . 5 6}$ \\
& MCR-Net-base & $\mathbf{8 3 . 9 7}$ & $\mathbf{8 1 . 2 2}$ & 86.83 \\
\hline \multirow{2}{*}{ DuReader } & RoBERTa-base & 19.35 & 11.87 & $\mathbf{5 2 . 3 8}$ \\
& MCR-Net-base & $\mathbf{3 4 . 2 2}$ & $\mathbf{2 8 . 0 4}$ & 43.91 \\
\hline
\end{tabular}

Table 3. Precision (P), Recall (R) and F1 of unanswerable questions on the two datasets for baselines and MCR-Net.

\begin{tabular}{l|cc|cc}
\hline \multirow{2}{*}{ Model } & \multicolumn{2}{|c|}{ SQuAD2.0 } & \multicolumn{2}{c}{ DuReader } \\
\cline { 2 - 5 } & EM & $\Delta$ & R-L & $\Delta$ \\
\hline Complete Model & $\mathbf{7 8 . 2 5}$ & - & $\mathbf{5 0 . 1 6}$ & - \\
\hline - Relation Block & 76.93 & -1.32 & 49.28 & -0.88 \\
- Stacked Relation Blocks & 76.05 & -2.20 & 48.35 & -1.81 \\
\hline
\end{tabular}

Table 4. Ablation study on model components.

the passage and question in a multi-step mode, and the performance can be boosted through this mutual information.

Performance of Unanswerable Questions. We evaluate the capability of the model to predict answerability in this section, results are described in Table 3 . The recall has improved significantly by $\mathbf{4 . 2 2} \%$ and $\mathbf{1 6 . 1 7 \%}$ on two datasets. It can be concluded that our model is more capable of finding unanswerable questions comparing with simple classifiers. And we explain why the question can not be answered by introducing the co-interactive relation module to explicitly locate key clues. Despite the decline in Precision, F1 score still has improved a lot. Another interesting finding is that the Recall on two datasets differs significantly. We make statistics of the number of unanswerable questions on two test results, and find that the number of unanswerable questions on the DuReader is really low $(5.33 \%)$. The analysis demonstrates that our method is more robust, however, the simple classifier has a poor performance on class imbalance problems.

Ablation Study. To get a better insight into our MCR-Net, we run the ablations on SQuAD 2.0 and DuReader, which is shown in Table 4. The stacked relation blocks make a contribution to the overall performance, which confirms our hypothesis that explicitly modeling mutual interaction and relation between the question and passage is important.

would take, we just use the large pre-trained language model in state-of-the-art comparison, while other results are based on the base model. We consider different steps of the interaction, the model performs best in the second step. So the results are reported with $s t e p=2$.

State-of-the-art Comparison. The main results are shown in Table 1 and Table 2 The proposed model consistently outperforms the previous methods and baseline models on two datasets. In SQuAD 2.0, 2.20\% gain on EM and $\mathbf{1 . 4 0 \%}$ gain on F1 on the ALBERT-base model, as well as improving on the ALBERT-xxlarge model. In DuReader dataset, our model can also make decent improvements. This result illustrates the effectiveness of explicitly modeling the interaction between

\subsection{Interpretability Study}

To demonstrate how the co-interactive relation module works when locating key clues, we conduct an interpretability study with the same example in Sec. 1. The attention maps of the first step and second step in the module are shown in Fig. 3. In the first step, the module attends some question-relevant clues such as later laws, opposition and so on. The plausible answer later laws is highlighted which is the wrong answer. And the module ignores the key clue little opposition. In Fig. 3 (b), we conclude that clues go from coarse to fine, 
and some coarse clues have been ignored, such as Migratory Bird Conservation Act of 1929. The wrong answer later laws has been corrected and the key clue little opposition is highlighted through multi-step co-interactive relation operation.

\section{CONCLUSION}

In this paper, we present the MCR-Net which explicitly models the mutual interaction between the question and passage and locates key clues from coarse to fine rather than in an implicit way. And we try to explain why this question is unanswerable instead of using a binary classifier that does not know the reason. We show that the proposed MCR-Net is effective and robust, which outperforms the previous methods with a single model, as well as improving the Recall and F1.

\section{ACKNOWLEDGMENTS}

This work is supported by the National Natural Science Foundation of China (No.62006222).

\section{REFERENCES}

[1] Karl Moritz Hermann, Tomás Kociský, Edward Grefenstette, Lasse Espeholt, Will Kay, Mustafa Suleyman, and Phil Blunsom, "Teaching machines to read and comprehend," in NIPS, 2015, pp. 1693-1701.

[2] Shuohang Wang and Jing Jiang, "Machine comprehension using match-lstm and answer pointer," in 5th International Conference on Learning Representations, ICLR 2017, Toulon, France, April 24-26, 2017, Conference Track Proceedings, 2017.

[3] Min Joon Seo, Aniruddha Kembhavi, Ali Farhadi, and Hannaneh Hajishirzi, "Bidirectional attention flow for machine comprehension," in ICLR, 2017.

[4] Ming Yan, Jiangnan Xia, Chen Wu, Bin Bi, Zhongzhou Zhao, Ji Zhang, Luo Si, Rui Wang, Wei Wang, and Haiqing Chen, "A deep cascade model for multidocument reading comprehension," in $A A A I, 2019$, pp. 7354-7361.

[5] Pranav Rajpurkar, Robin Jia, and Percy Liang, "Know what you don't know: Unanswerable questions for squad," in $A C L, 2018$, pp. 784-789.

[6] Wei He, Kai Liu, Jing Liu, Yajuan Lyu, Shiqi Zhao, Xinyan Xiao, Yuan Liu, Yizhong Wang, Hua Wu, Qiaoqiao She, Xuan Liu, Tian Wu, and Haifeng Wang, "Dureader: a chinese machine reading comprehension dataset from real-world applications," in $A C L, 2018$, pp. $37-46$.
[7] Xiaodong Liu, Yelong Shen, Kevin Duh, and Jianfeng Gao, "Stochastic answer networks for machine reading comprehension," in ACL, 2018, pp. 1694-1704.

[8] Christopher Clark and Matt Gardner, "Simple and effective multi-paragraph reading comprehension," in $A C L$, 2018, pp. 845-855.

[9] Minghao Hu, Furu Wei, Yuxing Peng, Zhen Huang, Nan Yang, and Dongsheng Li, "Read + verify: Machine reading comprehension with unanswerable questions," in $A A A I, 2019$, pp. 6529-6537.

[10] Ashish Vaswani, Noam Shazeer, Niki Parmar, Jakob Uszkoreit, Llion Jones, Aidan N. Gomez, L. Kaiser, and Illia Polosukhin, "Attention is all you need," ArXiv, vol. abs/1706.03762, 2017.

[11] Zhenzhong Lan, Mingda Chen, Sebastian Goodman, Kevin Gimpel, Piyush Sharma, and Radu Soricut, "ALBERT: A lite BERT for self-supervised learning of language representations," CoRR, vol. abs/1909.11942, 2019.

[12] Yinhan Liu, Myle Ott, Naman Goyal, Jingfei Du, Mandar Joshi, Danqi Chen, Omer Levy, Mike Lewis, Luke Zettlemoyer, and Veselin Stoyanov, "Roberta: A robustly optimized BERT pretraining approach," CoRR, vol. abs/1907.11692, 2019.

[13] Jacob Devlin, Ming-Wei Chang, Kenton Lee, and Kristina Toutanova, "BERT: pre-training of deep bidirectional transformers for language understanding," in NAACL-HLT, 2019, pp. 4171-4186.

[14] Oriol Vinyals, Meire Fortunato, and Navdeep Jaitly, "Pointer networks," in NIPS, 2015, pp. 2692-2700.

[15] Bishan Yang and Tom M. Mitchell, "Leveraging knowledge bases in lstms for improving machine reading," in Proceedings of the 55th Annual Meeting of the Association for Computational Linguistics, ACL 2017, Vancouver, Canada, July 30 - August 4, Volume 1: Long Papers, 2017, pp. 1436-1446.

[16] Fu Sun, Linyang Li, Xipeng Qiu, and Yang Liu, "Unet: Machine reading comprehension with unanswerable questions," CoRR, vol. abs/1810.06638, 2018.

[17] Kevin Huang, Yun Tang, Jing Huang, Xiaodong $\mathrm{He}$, and Bowen Zhou, "Relation module for nonanswerable prediction on question answering," CoRR, vol. abs/1910.10843, 2019.

[18] Zhuosheng Zhang, Yuwei Wu, Junru Zhou, Sufeng Duan, and Hai Zhao, "Sg-net: Syntax-guided machine reading comprehension," CoRR, vol. abs/1908.05147, 2019. 
[19] Yizhong Wang, Kai Liu, Jing Liu, Wei He, Yajuan Lyu, Hua Wu, Sujian Li, and Haifeng Wang, "Multi-passage machine reading comprehension with cross-passage answer verification," in ACL, 2018, pp. 1918-1927.

[20] Pranav Rajpurkar, Jian Zhang, Konstantin Lopyrev, and Percy Liang, "Squad: 100, 000+ questions for machine comprehension of text," in EMNLP, 2016, pp. 23832392.

[21] Chin-Yew Lin, "Rouge: A package for automatic evaluation of summaries acl," in Proceedings of Workshop on Text Summarization Branches Out Post Conference Workshop of ACL, 2004, pp. 2017-05.

[22] Kishore Papineni, Salim Roukos, Todd Ward, and WeiJing Zhu, "Bleu: a method for automatic evaluation of machine translation," in ACL, 2002, pp. 311-318.

[23] Diederik P. Kingma and Jimmy Ba, "Adam: A method for stochastic optimization," in 3rd International Conference on Learning Representations, ICLR 2015, San Diego, CA, USA, May 7-9, 2015, Conference Track Proceedings, 2015. 